Hernández-Merayo, E . (2013). Acampar sin permiso, pensar sin límite. Evaluación del proceso de un estudio de caso sobre el 15M (Granada). Revista Electrónica Interuniversitaria de Formación del Profesorado, 16 (3), 149-161.

DOI: http://dx.doi.org/10.6018/reifop.16.3.188491

\title{
Acampar sin permiso, pensar sin límite. Evaluación del proceso de un estudio de caso sobre el 15M (Granada)
}

\author{
Elisa Hernández-Merayo \\ Universidad de Granada
}

\section{Resumen}

En este texto se presenta el análisis reflexivo del proceso de una investigación, basada en un estudio de caso de la Asamblea de Plaza del Carmen (Granada)-15M; una investigadora participante se une y compromete con la asamblea durante el proceso que dura \#AcampadaGranada. Se presentan de forma simultánea el proceso de aprendizaje de una doctoranda que se introduce de pleno en el campo, participando activamente en él; y la evaluación de esta investigación orientada al campo social, sus potenciales y sus limitaciones.

\section{Palabras clave}

15M; reflexividad; investigación cualitativa; investigadores noveles.

\section{Assemble without permission, think without limits Process evaluation of a case study about the 15M (Granada)}

Contacto:

Elisa Hernández Merayo, ehm@ugr.es, Facultad de Ciencias de la Educación, Campus Universitario Cartuja, Universidad de Granada, 18071 Granada. Tel: 958243742

Investigadora financiada por una beca FPU AP2009-2243 


\section{Abstract}

This paper is about an evaluation of a reflective research process - a Case Study about Plaza del Carmen Assembly (Granada, Spain); 15M- from the point of view of Education. A participant researcher join and commit herself with the Assembly process. On one hand we present the learning process of a doctoral student who started her field work immersed in the Assembly, actively participating in it. In the other we present an evaluation of this social change oriented research, with its possibilities and limitations.

\section{Key words}

15M; reflexivity; qualitative research; junior researchers.

\section{Introducción}

La primavera de 2011 vino llena de sorpresas que siguieron al primer asombro provocado por una masiva afluencia a la manifestación del domingo 15 de mayo convocado por la plataforma ciudadana Democracia Real Ya. En Granada, ciudad del estudio, también fuimos sorprendidos. Fue una sorpresa marcada por una convocatoria en un momento estratégico previo a las elecciones municipales, que tuvo la sabiduría de no posicionarse de cara a las mismas; marcada porque dejó constancia de una multitud de la ciudadanía que pensaba de forma alternativa ante la cual la minoría política hacía tiempo que había vuelto la cabeza; por la aparición de numerosos nuevos agentes sociales; y por el surgimiento de visiones diferentes de la política que se acercaban a esta de un modo amplio y emergente, de una política de y para todas y todos.

Supuso no sólo una sorpresa individual de aquellos nostálgicos y nostálgicas del mayo del 68 y una sorpresa social que alentó la esperanza de quienes piensan que "otro mundo es posible" y puso en vilo a la minoría que no desea modificar el status quo en aras a mantener su posición privilegiada; sino también una sorpresa mediática para periodistas que no encontraban interlocutores, instituciones que no encontraban portavoces con quienes negociar, y policía que no encontraban líderes que acallar; y una sorpresa urbana marcada por la emergencia, una acampada que alteraba el estilo de retirada de la vida pública impuesto por la ordenanza cívica de Granada.

La gran sorpresa no vino únicamente determinada por la cantidad de personas y de propuestas, sino también por el contenido de esas propuestas y peticiones, por el lugar desde donde se pedían y por las formas en que estas se negociaban.

Sorprendidas nosotras y nosotros también, desde el grupo "Investigación Curricular y Formación del Profesorado" (ICUFOP), sentimos la necesidad de acercarnos a esta nueva realidad emergente, renovada y potente para aprender de ella. Lo hicimos desde el proyecto "La ciudadanía en los nuevos escenarios digitales y escolares: relaciones $e$ implicaciones en el alumnado de la Educación Secundaria Obligatoria (ESO)", cuyo objetivo es identificar el "ethos" democrático entendido como vivencia, experiencia o sentimiento de las y los jóvenes al relacionarse y comunicarse con las y los demás, como orden simbólico que facilita reglas para regular el comportamiento que es deseable o el que no lo es.

Pensando en la asamblea de Plaza del Carmen (Granada) - para saber más sobre la Asamblea de Plaza del Carmen, como por ejemplo su consenso de mínimos o las demandas urgentes, puede visitarse http://acampadagranada.org - como forma de vivir y construir la democracia, como proceso de aprendizaje político, como lugar de experiencia de la 
ciudadanía, como espacio de empowerment y desarrollo de la agencia y el compromiso cívico-político; no podíamos dejar de mirar hacia la plaza como un espacio privilegiado para comprender la construcción del ethos democrático, entendido como producto social aprendido vivencialmente, como modo de vida, fundamento de praxis, raíz de la que emergen las acciones humanas. Es por ello que decidimos recoger el proceso de construcción de ciudadanías de la Plaza del Carmen a través de un estudio de casos, cuya discusión como investigación socialmente comprometida introducimos en el presente artículo, revisando especialmente el proceso de investigación con la finalidad de enriquecerlo y reflexionar sobre sus puntos positivos y sus debilidades.

Se trató de una investigación emergente, por lo que partiendo del estudio de casos como herramienta metodológica, fuimos adaptándonos al impredecible devenir de las asambleas, adaptándonos a ellas. Por lo que no empleamos ningún protocolo rígido y estable, sino que la flexibilidad dominó el estudio y a mediante ella fuimos adaptándonos a nuestros focos de interés a través de una mirada siempre educativa: la ciudadanía, la construcción de la política, los y las jóvenes, los nuevos medios de comunicación.

En el periodo de inmersión en campo se une la investigación vinculada al cambio social con mi propio proceso de formación como investigadora y una socialización política profunda, cobrando para mi un sentido muy especial, que sin duda ha marcado mi trayectoria personal y profesional. Por tanto, en estas líneas pretendo reflexionar sobre cómo se vincula la implicación social orientada al cambio a la formación de investigadoras e investigadores noveles.

\section{Desarrollo}

Los objetivos se han dirigido a comprobar cómo los y las jóvenes sienten, viven y experimentan la ciudadanía democrática, participando en el lugar público de la plaza y en las diferentes esferas virtuales -libres y comerciales- donde se está formando una más informada ciudadanía entre la convergencia del debate público, la hipertextualidad y la no linealidad.

Puesto que esta fue nuestra primera entrada en el campo, en la maleta sólo llevábamos las discusiones previas sobre las diferentes nociones teóricas que abordamos desde el proyecto; entendiendo la política en su amplio sentido de intereses encontrados, la ciudadanía como la forma de vivenciar el ethos democrático, la juventud como agentes activos en búsqueda de su emancipación y las nuevas tecnologías de la información y comunicación como potenciales canales de comunicación y debate en los que se se abre el espacio red, donde se puede llegar a generar una esfera pública virtual. Discusiones y nociones previas aun por completar, re-definir y comprender a partir de la inmersión de los procesos prácticos reales, pero que son necesarias de compartir puesto que afectan a nuestra forma de acercarnos al campo (Optamos por la explicitación de la ideología y pre concepciones en lugar de considerar a las y los investigadores como tabulas rasas)

La metodología que entendimos como más apropiada fue el estudio de casos, debido a la unicidad de la asamblea que deseábamos comprender en profundidad, siguiendo la metodología de Stake (2007) y de Simon (2011).

Dado el carácter emergente de las asambleas (que impidió establecer un protocolo a priori), y mi progresiva formación práctica a partir de la inmersión en el campo, toma especial relevancia el proceso de investigación, sobre el cual fuimos tomando las decisiones que estimamos más pertinentes. 


\section{Proceso de investigación}

La investigación se inicia de forma incidental el 17 de mayo en la primera asamblea ciudadana convocada a través de las redes sociales. Acudí a ella por una conciencia personal que me inclinaba hacia el trabajo por un cambio social tan necesario en estos momentos de retroceso de los derechos ciudadanos y la justicia social. En ella comprendí que algo importante comenzaba a surgir, y que estaba vinculado estrechamente con la construcción de la ciudadanía y la política, eje principal del proyecto I+D en el que estábamos inmersos y de mi proyecto de tesis. Contacté esa misma tarde con el responsable del grupo de investigación y director de tesis, Juan Bautista Martínez Rodríguez, y decidimos embarcarnos en este proceso a través de un estudio de casos.

Con un kit etnográfico improvisado (varios bolis, un cuaderno, y una cámara fotográfica con apenas batería - que fue tecnologizándose posteriormente con los recursos del grupo: grabadora y cámara de video) comencé esta investigación emergente desde esa misma noche. Me implique desde sus inicios en esta asamblea siguiendo sus pasos día y noche durante los 32 días que duró la acampada; la observación participante (o más adecuadamente, participación observante) fue registrada en un cuaderno de campo al que se vinculaba un pequeño diario de investigadora en el que iba recogiendo los cambios que iba percibiendo en mis propios procesos. Las observaciones de este cuaderno de campo fueron afinándose cada vez más, siendo en un principio toscas y en ocasiones entremezcladas con mi propia vivencia; poco a poco fui avanzando en la difícil tarea de la observación y descripción (el proceso de aprendizaje como investigadora es fácilmente perceptible al observar la evolución del cuaderno de campo y las reflexiones del diario de investigadora).

Los constantes diálogos que mantuve con Juan Bautista y su permanente apoyo fueron para mi las claves que me ayudaron a avanzar en el proceso de investigación y a mantener una constante reflexividad. Tras la primera semana de observación "bruta" del contexto, junto con Juan establecimos el primer borrador de categorías de análisis sobre las que se focalizaría la observación, ejes o issues, que fueron modificándose a lo largo de la investigación a medida que esta lo requería: "tabla 1: ejes temáticos" 


\section{Tabla 1. Ejes Temáticos}

\section{a. Ejes temáticos}

- Comunicación: construcción de la realidad.

- Lo público en los medios de comunicación

- Medios independientes: radio Plutón (entrevista), público, etc.

- Redes sociales: n-1, Facebook, twitter, Tuenti, blog (tomalacalle.net). Papel de la comisión de redes, relación con otras acampadas

- Prensa: local (Granada hoy, Ideal), nacional / versión impresa y versión digital. Periódico propio: Ágora.

- Espacio público (público Vs privado / recuperación del espacio público):

- La plaza: desalojo, lo público en lo privado y lo privado en lo público, patrones de ocupación del espacio, actividades y espacio, espacios privilegiados. Acción contra la banca: asamblea en un bando

- Manifestaciones: 15M, 9J, 19J (convocadas por el movimiento) + manifestación LGTB, manifestación parados, manifestación por el pueblo sirio, manifestación contra despidos de telefónica

- Desalojo del CSO la Indiskreta

- Lo político:

- Concepciones políticas:

- Discurso de lo político: asamblea, apolítico Vs apartidista, asindicalista, aconfesional, representatividad, voto Vs consenso, etc.

- Democracia

- Ciudadano:

- Juventud Vs otras generaciones

- Infancia

- Inmigración

- Sin hogar y personas con trastornos mentales.

- Relaciones institucionales/con la política pública:

- Subdelegación del Gobierno

- Ayuntamiento

- Universidad (posición del decano y los rectores, "toma la Uni")

- Con la política institucional:

- con los partidos políticos PP, PSOE, IU, IA

- Continuo anti-sistema - altersistema

- $\quad$ relaciones entre política institucional y política no institucional

$\checkmark$ Relaciones con las políticas privadas:

1. Banca

2. Multinacionales: "información libre con nombres y apellidos"

3. Empresas locales: bares, comercios y asociaciones de comerciantes

$\checkmark \quad$ Participación y organización

- Evolución de la estructura del movimiento: asambleas, megafonía, infraestructura, etc.

- Evolución de los grupos de trabajo: cómo ha crecido y por dónde (grupo de coordinación)

- Proceso de aprendizaje:

- Aprender a relacionarse con las políticas institucionales

- Aprender el proceso de asamblea.

- Relaciones con otras asambleas

- Relación entre grupo de trabajo (grupo interasambleas)

- Relación con otras ciudades (reunión en Sol)

- Relación con las asambleas de barrios y Universidades

- Relación con Democracia Real Ya

\section{b. Ejes transversales}

- Género:
- Distribución en el espacio
- Intervenciones y usos de la palabra, las propuestas y sus desarrollos
- Componentes de los distintos grupos de trabajo
$\circ \quad$ Lenguaje 
Al cuaderno de campo y diario de investigadora se unieron la recogida de documentos (actas, materiales elaborados por los grupos de trabajo de la asamblea, los número de Ágora el periódico de la asamblea, etc); un sinfín de material fotográfico y vídeos de eventos más destacables. $Y$ varias entrevistas informales realizadas en el mismo campo.

Los momentos y giros de la investigación fueron marcados por el devenir de la asamblea:

1. primera asamblea en en el paseo del salón y decisión de acampar en la plaza del ayuntamiento

2. Inicio de \#AcampadaGranada, primeras asambleas organizativas y grupos de trabajo, coon posterior desalojo por la policía en la madrugada - "hoy somos cien mañana seremos mil"

3. Incremento masivo de las asambleas y grupos de trabajo

4. Comienzo de la difusión en las facultades

5. Aprendizaje de cómo compartir el espacio público- primera coincidencia de un acto ciudadano programado en la Plaza del Carmen, esta vez por un colectivo LGTB apoyado por sindicatos

6. Jornadas de reflexión de elecciones municipales

7. Desalojo del CSO La Indiskreta que había proporcionado apoyo logístico a la acampada

8. Acto de investidura del alcalde

9. Difícil decisión del levantamiento de la acampada- "no nos vamos de la plaza, tomamos la ciudad".

Estos son algunos de los eventos más destacados, junto con la continua evolución de los grupos de trabajo, la cambiante distribución y uso del espacio público, y la progresiva descentralización de la Asamblea hacia asambleas de barrio y de Universidad.

Paralelamente a mi trabajo en el campo, Juan Bautista fue recopilando la prensa local y nacional relativa al tema; y Maria del Carmen Robles, otra investigadora del grupo, siguió algunas cuentas de Twitter vinculadas a la acampada. Así mismo, recuperé información del perfil de Facebook de la asamblea aunque este análisis nunca llegó a abordarse.

Con un amplio volumen de información por analizar, el levantamiento de la acampada puso fin a la recogida de datos (aunque no a mi implicación en la asamblea); y pasamos a dedicarnos al análisis, aunque este ya había ido acompañando en cierta manera todo el proceso de campo.

El análisis y la interpretación de los datos se hicieron bajo los criterios cualitativos de dar la voz a los grupos singulares, subjetividad crítica, reflexividad del investigador y la reciprocidad (Simon, 2011: 181).

Juan Bautista Martínez (director del proyecto y director de mi tesis) analizó la prensa (principalmente Granada Hoy, Ideal, Ágora -el periódico propio de la asamblea- y los diferentes comunicados emitidos por la asamblea), Carmen Robles los twits recogidos, y yo analicé el diario de cuaderno de campo y los materiales documentales recogidos, junto con el diario de investigadora (que se inicia en este momento pero continuará abierto hasta el final de mi tesis donde realizaré un análisis global de mi propia trayectoria). Inicialmente pretendimos construir la historia simultáneamente a partir de una wiki creada por Enrique Díez, pero la densidad de los datos dificultó el uso de la misma por su lentitud; además de que no conseguimos argumentar nuestros tiempos para que la wiki se convirtiese en un 
espacio verdaderamente dialógico entre las y los investigadores. Finalmente cada uno realizó su análisis para posteriormente triangularlo.

En un intento de triangulación de los investigadores (presentación de las observaciones e interpretaciones a otro investigador para analizar interpretaciones alternativas- Stake 2005) simultanea a la triangulación de fuentes de datos, Feliciano Castaño trató de cruzar los datos e interpretaciones de los tres investigadores para la realización del informe final; pero la distancia del campo, la diferente postura epistemológica, y las dificultades de enfrentarse a análisis parciales muy diversos dificultó en exceso su tarea.

Tras este intento de triangulación doble fallida finalmente fui yo - que por aquel entonces ya me había separado bastante del campo, aconteciendo una estancia entre medias y pasado casi un año completo) quien realicé la triangulación de las fuentes de datos. Junto con la ayuda de los diálogos con Juan Bautista integramos los tres análisis. Quizás por que a mi me era más sencillo hacer una composición de lugar al tener mucha más información conservada en la cabeza, que por unos u otros motivos (entre ellos mi inexperiencia), no había sido plasmada en los análisis previos -e incluso tampoco en los materiales brutos- y sin embargo era clave para la triangulación. Finalmente las primeras categorías iniciales flexibles se convirtieron en el siguiente esquema:

Tabla 2. Sugerencias pedagógicas del $15 \mathrm{M}$

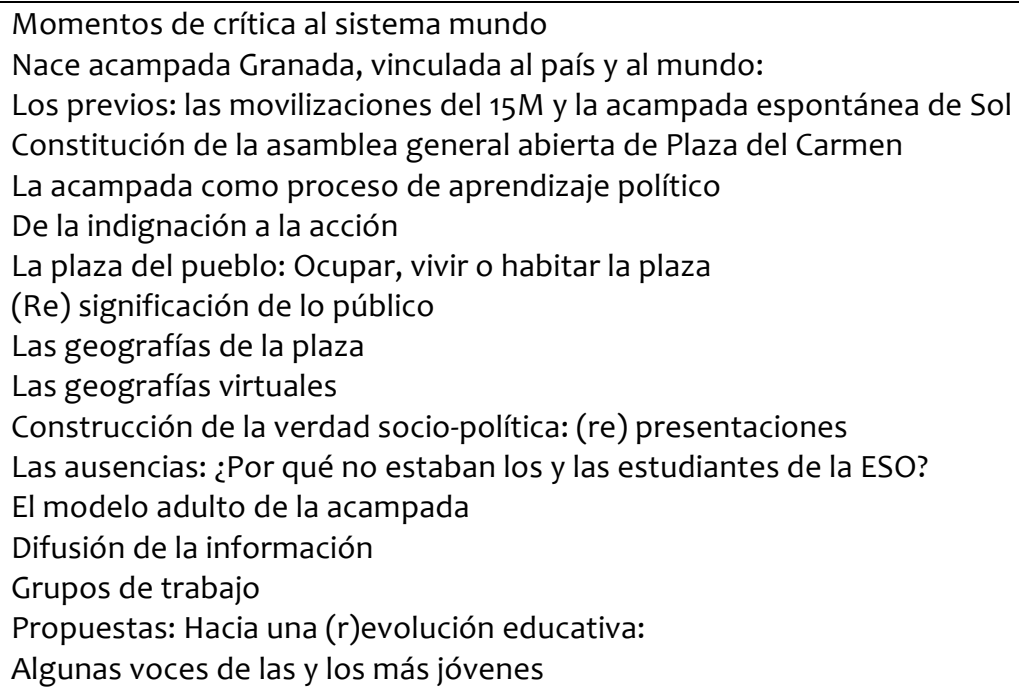

Finalizado el informe hubiese sido necesaria su devolución y la negociación del mismo con la asamblea, pero esta como tal estaba pasando un periodo bajo y tratando de reorganizarse por si misma, puesto que sus fuerzas habían dado lugar a otros grupos de activismo fuertes (como el grupo stop desahucios, stop represión o las cooperativas de agricultura locales). Digamos que en cierto sentido la asamblea estaba en un periodo de grandes cambios.

La difusión de la información se realizó a través de varias comunicaciones, en entornos más o menos formales, y publicaciones en diversos espacios. Así mismo, consideramos las redes sociales como un medio de difusión del mismo que llega a más personas que se encuentran fuera del entorno académico. 


\section{El cambio}

El análisis de los resultados es denso y extenso por lo que no pretendo aquí más que hacer una introducción a los mismos y dedicar el espacio a la reflexión sobre el proceso y los límites (Puede accederse al informe completo en http://elisamerayo.wordpress.com/2013/06/16/informe-caso-asamblea-granada-15m/ ):

En la asamblea nos encontramos con un crisol de heterogeneidades, en especial en relación a su trayectoria activista previa (desde quienes dieron sus primeros pasos en la plaza hasta quienes llevaban una larga trayectoria y experiencia militante) y a su postura ideológica (especialmente en las primeras propuestas recogidas que mostraban una rica heterogeneidad:

¿Por qué el 15M se convocó para 300 personas y fuimos 10.000? primero porque ellos nos han sacado a la calle, tenemos distintas ideas pero nos han unido porque nos han tomado a todos el pelo. Lo único que nos une es que salgamos con nuestra propia voz según aquello que tenemos todos en común. No dejéis que nadie os pida el voto haciendo uso de lo que se hizo en el $15 \mathrm{M}$ y lo que se hace aquí. (Extracto de la asamblea 18 de mayo)

Aunque esta fue evolucionando hacia una ideología que pretendía de una u otra forma romper con el status quo del sistema, oscilando entre el continuo altersitema- anti sistema: sitema antihumano, humano antisistema; No somos antisistema; el sistema es antinosotros; fallo del sist€ma, resetear; otro sistema es posible (pancartas de la plaza)

Aunque había presencia de todas las edades, destacaba una clase media de jóvenes con estudios superiores o cursándolos, que se ve envuelta en un proceso de desclasamiento y se ven "sin futuro- $\sin$ casa, sin curro, sin miedo". Una juventud que se reclama a sí misma como ciudadanía activa, en contra de la opinión generalizada y trasmitida a través de los medios de comunicación, jóvenes que encuentran en la plaza un lugar para la emancipación liberadora (Kushner, Wattie, Kutanegra 2011). Nos cuestionamos por qué no estaban presentes jóvenes de menor edad, por lo que al concluir el estudio de caso realizamos dos entrevistas a jóvenes de la ESO que nos expusieron su visión de la asamblea y los motivos por los que no se implicaron activamente en ella. Así mismo, realizamos un estudio exploratorio en Tuenti - red social usada mayoritariamente por ellos.

"Antes de que se vayan los medios de comunicación quiero decir que se dice que los jóvenes estamos dormidos, que no hacemos nada, que estamos descomprometidos; se equivocan. Quiero decir a los políticos que no son de otra clase (...) como juventud española queremos poder pagar nuestras pensiones" (Extracto de la asamblea del 26 de mayo)

La crítica gira en torno a tres ejes: la clase política, los bancos y los medios de comunicación de masas (extendiéndose en algunos casos a la policía como represores de los movimientos sociales), que se consideran como causantes de la crisis política, social, económica y ética en la que estamos inmersos - "no somos mercancias en manos de políticos y banqueros", "Medios de des-comunicación de masas" (Pancartas)

La crisis de la representatividad (la democracia representativa y los medios de comunicación como creadores de las representaciones de los imaginarios sociales) da paso a un diálogo deliberante. Un diálogo basado en consensos que avanza hacia la democracia deliberativa, basada en la esfera pública de la plaza y extendida hacia la esfera virtual; que hace uso de los nuevos medios para la comunicación sin intermediarios, la información alternativa y el debate (empleando redes sociales en ocasiones libres y en ocasiones comerciales) (Sádaba y Gordo, 2008) 
"nos vemos aquí, nos vemos en internet, nos vemos en radio Plutón" (Extracto de la asamblea del 18 de mayo)

Las y los activistas se re-apropian del concepto de política, sacándolo de las paredes del ayuntamiento, entienden la política desde las relaciones diarias, desde lo cotidiano y lo local; una política de lo público y lo privado, ampliando las formas de lo decible y modificando las condiciones formales de participación (Moreno Pestaña, 2011), tanto en la plaza como en la red; una política que rejuvenece y se llena de acciones creativas y lenguaje irónico sin ser ingenua, sin abandonar los debates políticos profundos. A modo de ejemplo (tan sólo uno entre muchos), podemos citar las performances realizadas en dos sucursales bancarias del centro de Granada: http://vimeo.com/24391763

A modo de conclusión general abreviada (para encontrar las conclusiones elaboradas Hernández, Robles y Martínez, 2013) destacamos que las escuelas- envueltas en la tensión entre la reproducción y la salvación escolar (Martín Criado 2010) pueden aprender mucho de los movimientos sociales, pues tanto plazas (físicas y virtuales) como escuelas son espacios privilegiados para la vivenciar la autonomía, ensayar la agencia política, experimentar la democracia, y aprender a comunicarse, a disentir de la autoridad, y a construir aprendizaje colectivo con sentido socio-político. Así mismo, la política necesita tanto de la pedagogía para llegar a todas y todos, como la pedagogía necesita de la política puesto que ninguna decisión educativa carece de ideología.

\section{Discusión sobre los límites de la investigación}

Uno de los límites que encontramos fue debido a mi inexperiencia y excesiva identificación con la asamblea, lo que dificultó el análisis en cierto sentido y requirió un trabajo extra de distanciamiento y separación de mi yo activista y mi yo investigador. Al mismo tiempo que supuso una experiencia política imprescindible para mi crecimiento personal requirió múltiples conversaciones con personas ajenas al campo, en especial con Feliciano Castaño (compañero del grupo de investigación) y Juan Bautista, para aprender a discernir. Una de las propuestas interesantes que se sugirieron para abordar esta dificultad (aunque de momento no se han llevado a cabo), fue la creación de un personaje para mi "yo activista", una especie de alter ego que bajo un pseudónimo narrase la experiencia en primera persona, la experiencia de la agencia dentro del campo, un personaje que pudiese debatir con mi "yo investigador" que teoriza sobre la práctica emergente en función de una teoría fundamentada. En ningún momento estuvo previsto que renunciase a mi experiencia política y compromiso social que tan necesaria me resultaba para mi crecimiento personal y para dar completo sentido a los conceptos que abordo desde mi tesis, aunque esto retrasase el proceso de la investigación.

La falta de organización en ocasiones supuso una barrera entre un equipo mayor de investigadores que en principio íbamos a abordar el tema y quienes finalmente realizamos la investigación. Sin duda, nos costo articular las distintas motivaciones y niveles de compromiso con el tema. En especial supuso un necesario aprendizaje de articulación de tiempos que oscilaba entre la urgencia de una investigadora dedicada al campo (en continua evolución) en exclusiva y los tiempos lentos de la academia y sus múltiples responsabilidades ajenas al tema.

Tratamos de abordar las distancias físicas (el equipo investigador en principio pertenecía a diferentes Universidades españolas) y temporales mediante el empleo de la wiki anteriormente comentada, pero no nos sirvió para acomodar los distintos ritmos de trabajo. Sin embargo, otras y otros investigadores han empleado esta estrategia de forma efectiva abordando OccupyWallStreet/OccupyTogether, a partir de la wiki OccupyResearch, superando con creces nuestras expectativas sobre la herramienta y articulando el trabajo 
de investigadoras e investigadores que "compartieron ideas, preguntas de investigación, métodos, herramientas, bases de datos; para después trabajar en la recopilación, análisis, discusión, escritura, etc. para desarrollar teoría y práctica sobre el movimiento Occupy" (http://occupyresearch.wikispaces.com). En España el proyecto "colaborativo, copyleft, transmedia y sin ánimo de lucro" $15 \mathrm{M} \mathrm{cc,} \mathrm{del} \mathrm{"laboratorio} \mathrm{del} \mathrm{pro-común"} \mathrm{de} \mathrm{mediaLab}$ Prado, empleo la wiki para recoger diferentes miradas al 15M; aunque la parte destinada a proyectos de investigación es muy escaso y se limita a compartir papers (http://papers.15m.cc/). Por tanto consideramos, que la herramienta de la wiki es un canal de comunicación que ofrece muchas posibilidades para la investigación colaborativa, sin embargo este tipo de colaboración intensa requiere un aprendizaje previo. Es un canal de comunicación muy útil pero no soluciona los problemas de ajuste previos.

Otra de las limitaciones está asociada al tiempo que tardó la investigación en finalizarse y los lentos canales de distribución que se emplearon para difundir la experiencia, comparados con el vertiginoso ritmo de evolución de la asamblea (en parte relacionados con el ritmo de la academia y en parte por problemas organizativos, sin olvidar los personales que me alejaron de la investigación por un tiempo debidos a la necesidad de distanciarme para poder re-analizar desde un punto más distante); considero que hubiese resultado más útil poder haber realizado una devolución a la propia asamblea, aunque finalmente no pudo ser posible.

Una cuestión a tener en cuenta es la velocidad de producción de las diferentes redes sociales, por tanto es esencial delimitar cuidadosamente el campo y seleccionar la información más relevante. Hubiese sido interesante recoger los primeros twits y poder observar la gestación y negociación de la asamblea también en la red; pero tomamos la decisión a posteriori y la asamblea, junto con la acampada, llevaba ya 15 días emitiendo información, por tanto hemos de situar cronológicamente esos twits en su momento de producción, y considerarlos como las comunicaciones emitidas por una asamblea ya consolidada.

Finalmente cabe resaltar, que quizás por mi inexperiencia (y el primer desasosiego por recoger todo cuanto acontece, tal vez por miedo a quedarse corto en los datos para hallar una comprensión, tal vez por la ansiedad que genera desprenderse del campo para pasar a dedicarse en exclusiva al análisis) o quizás por falta de organización rápida en un proceso emergente, se recogió un excesivo volumen de información y no toda pudo ser analizada, al menos para ese primer informe. Dedicamos excesivo tiempo en recoger información del Facebook que nunca fue analizada; y el amplio volumen de material fotográfico y audiovisual aun no ha sido terminado de analizar aunque proseguimos con ello aun a riesgo de llegar tarde.

\section{Propuestas}

Los contratos de investigación con la propia comunidad a estudiar son uno de los compromisos éticos que solemos seguir a la hora de abordar la investigación social y educativa, sin embargo dadas las características de la asamblea, multitudinarias en un primer momento fue imposible de realizar. (Sin embargo, nunca se negó la presencia de la investigadora - y activista- en el campo, y fueron contestadas todas las preguntas sobre la investigación que fueron realizadas por el resto de activistas, no encontrando resistencia por ninguna de las personas que preguntaron)

Otro aspecto que siempre hemos considerado imprescindible, es el proceso de devolución a los y las propios agentes de la investigación y la negociación de los informes producidos. 
Aunque como mencionábamos anteriormente esta ha sido una de las grandes limitaciones de nuestra investigación, cuando el informe estuvo elaborado, muchas de las y los agente implicados ya no estaban presentes en la asamblea y la negociación resultó imposible en el seno de la misma, que hubiese sido su lugar.

Dado que hemos empleado el estudio de casos, nuestra pretensión siempre ha sido profundizar en un contexto local determinado, limitado; es decir hacer un corte en la realidad y profundizar en la comprensión del mismo. Los resultados por tanto no son extrapolables a otras situaciones y contextos similares. En nuestro caso, hemos abordado el estudio de la asamblea de Plaza del Carmen (Granada), no hemos pretendido abordar la globalidad del movimiento $15 \mathrm{M}$ en la que esta se encuentra inmersa (aunque lógicamente hemos considerado el contexto de la misma); por tanto nuestras conclusiones no son válidas para el movimiento sino para la asamblea. En este sentido, es de vital importancia mantener claro los límites del campo que se está estudiando.

Es necesario mantener presente que las miradas y perspectivas desde las que se aborda la comprensión de un movimiento social no son únicas, no existe una verdad única y completa, y cabe la discrepancia (que a ser posible es necesario recoger). Si admitimos la diversidad ideológica y de paradigmas presentes en la plaza, resultaría absurdo negar esa diversidad a la hora de acercarnos a esa realidad plural a través de la investigación. Por tanto, este estudio de casos no pretende ser una lectura exclusiva de la Asamblea de la Plaza del Carmen, sino tan sólo una de entre las múltiples posibles; una interpretación que pretende ser una mirada desde el punto de vista educativo dadas las características de nuestra formación y función del grupo de investigación.

A partir de nuestra propia experiencia, creemos que una investigación colaborativa es mucho más enriquecedora para el campo que una mera investigación individual puesto que a partir de las discusiones sobre los diferentes sentidos posibles y perspectivas plurales se construye una mirada más completa. Aprender a investigar de forma verdaderamente colaborativa se convierte en una necesidad en la sociedad de redes. Internet puede aportar nuevas herramientas y canales de comunicación para trabajar en el espacio de flujos (Castells, 2009) cuando participan investigadores de distintas universidades, pero sin olvidar que la base del proceso es el diálogo (y estas herramientas y canales solo resultan útiles si se emplean de la manera adecuada). En este sentido, la academia española tiene mucho que aprender sobre los usos que los movimientos sociales hacen de los nuevos medios alternativos que surgen en la red (Sádaba y Gordo, 2008).

En este tipo de investigaciones orientadas al cambio social que requieren un alto grado de implicación se produce una transformación tanto en la comunidad estudiada como en la investigadora. Desde este punto de vista consideramos que es importante recoger también los cambios producidos en las investigadoras o investigadores, a través de un diario de investigadora; aunque consideramos que la plasmación de este proceso es siempre importante, creemos que se vuelve imprescindible cuando se trata de investigadoras noveles, puesto que necesitamos una constante reflexividad para hacer conscientes los procesos que nos marcarán como primeras experiencias formativas y serán en cierto sentido determinantes en nuestro estilo de investigación posterior.

Unida a la reflexión anterior consideramos que en este tipo de investigación es necesaria una cierta coherencia, es decir, no es posible tratar de producir una investigación orientada al cambio si nosotros no estamos dispuestos a cambiarnos por dentro, a aprender del campo, a evolucionar junto con el resto de agentes implicados; al igual que no es posible investigar sobre ciudadanía y democracia anti-democráticamente. En este sentido debemos 
ser investigadores flexibles capaces de ajustarnos a los procesos de la propia práctica, y por que no decirlo, capaces de apasionarnos con la búsqueda de sentidos compartidos.

\section{Bibliografía}

Castells, Manuel (2009) Comunicación y poder. Madrid: Alianza Editorial.

Hernández, Elisa; Ma Carmen Robles y Juan Bautista Martínez (2013) Jóvenes interactivos y culturas cívicas. Sentido educativo, mediático y político del 15M. Comunicar, 40 (5967)

Martín Criado, enrique (2010) La escuela sin funciones. Crítica de la sociología de la educación crítica. Barcelona: Bellaterra.

Moreno Pestaña, Jose Luis (2001) Pensar la palabra libre con Michael Foucault. Una etnografía de las asambleas del 15M. Pasajes: revista de pensamiento contemporáneo. 36 (89-99)

Sádaba, Ígor y Gordo, Ángel (coords.) (2008). Cultura digital y movimientos sociales. Madrid: Catarata.

Simon, Hellen (2011). El estudio de caso: Teoría y práctica. Madrid: Morata.

Stake, Robert (2005). Investigación con estudio de casos. Madrid: Morata.

Kushner, Saville; Anna Marie Wattie y Made Kutanegra (2011). Evaluación, adolescentes y derechos. Revista Interuniversitaria de Formación del Profesorado, 71 (25.2) 


\section{Autor}

Elisa Hernández Merayo

Maestra en educación infantil, Licenciada en Psicopedagogía, Máster en Estudios Migratorios, Desarrollo e Intervención Social. Actualmente cursando el Doctorado en Ciencias Sociales Aplicadas. Becaria FPU del departamento de Didáctica y Organización Escolar de la Universidad de Granada y miembro del grupo Investigación Curricular y Formación del Profesorado (ICUFOP) -HUM267 